\title{
Role of Immunohistochemistry in The Subtyping of Non Small Cell Lung Carcinoma on True Cut Lung Biopsies
}

\author{
Alekhya. $\mathbf{M}^{1}$, Rukmangadha. $\mathbf{N}^{1 *}$,Lakshmi.AY ${ }^{2}$, Manickavasagam. $\mathbf{M}^{3}$ \\ ${ }^{1}$ Department of Pathology, Sri Venkateswara Institute of Medical Sciences, Tirupati, India \\ ${ }^{2}$ Department of Radiology, Sri Venkateswara Institute of Medical Sciences, Tirupati, India \\ ${ }^{3}$ Department of Medical Oncology, Sri Venkateswara Institute of Medical Sciences, Tirupati, India
}

\begin{abstract}
Background: Worldwide, lung carcinoma is the most common cancer in terms of number of cases and deaths. Lung carcinomas are broadly divided into small cell carcinoma and non-small cell lung carcinoma(NSCLC). In recent years availability of targeted therapies necessitated subtyping the NSCLC to improve the survival and quality of life. NSCLC can be subtyped by routine Haematoxylin and Eosin (H\&E) stained section slides alone, poorly differentiated tumors are difficult to segregate morphologically, especially in true-cut biopsies, necessitating ancillary techniques like immunohistochemistry (IHC). Hence this study was taken up to examine accuracy of diagnosis of Non-Small Cell Lung Carcinoma (NSCLC) on biopsy samples initially made based on morphology and then with IHC using relevant markers like CK5, CK6, CK7, Napsin-A, TTF-1, P63, Synaptophysin and Chromogranin-A.
\end{abstract}

Methods: A prospective study of two years and six months duration during which 111 cases of NSCLC on true-cut biopsies were first reported on Haematoxylin and Eosin sections and later subjected for IHC.

Results: Outof 111 cases, after IHC, 80 were diagnosed as adenocarcinoma and 31 as squamous cell carcinoma NSCLC were common in the 6th decade. In adenocarcinoma positivity for CK7, TTF1 and Napsin-A was $95 \%, 75 \%$ and $78.75 \%$ respectively. In squamous cell carcinoma positivity for CK5,CK6and P63 was $84 \%, 81 \%$ and $90 \%$ respectively.

Conclusion: CK5, CK6 and P63 can be used for confirming squamous cell carcinoma and CK7, TTF-1 and Napsin-A for adenocarcinoma. We recommend a IHC panel consisting of P63, TTF1 and Napsin-A in NSCLC-unclassifiable and poorly differentiated carcinoma on H\&E.

Keywords: Adenocarcinoma, Squamous Cell Carcinoma, CK7, CK5, CK6, P63, Napsin-A, TTF1

\section{Introduction}

Worldwide, lung carcinoma is the most common form of cancer in terms of number of cases and deaths, accounting for $13 \%$ of all new cases and $19 \%$ of cancer related deaths with majority of deaths now occurring in developing countries $(55 \%)$. ${ }^{[1,2] . ~ S i n c e ~ t h e ~ p a s t ~} 2$ decades adenocarcinoma is observed to be the most common type of lung cancer compared to squamous cell carcinoma. ${ }^{[3]}$ Lung carcinomas are broadly classified into small cell carcinoma (20\%) and non-small cell lung carcinoma(NSCLC) (80\%). The main histological subtypes of NSCLC are squamous cell carcinoma, adenocarcinoma and large cell carcinoma. Earlier, the histologic sub-classification was not clinically or therapeutically important and all subtypes received the same treatment. In contrast in the past few years the availability of targeted therapies has made the need for subtyping the NSCLS so as to improve the survival and quality of life. Hence, a new classification has been proposed by the IASLC/ATS/ERS for small biopsies and cytology samples which emphasizes the use of histochemical (mucin staining) and immunohistochemical (IHC) stains and molecular studies apart from routine histomorphology on Haematoxylin and Eosin (H\&E) stained slides. Though majority of the NSCLC can be subtyped by routine H\&E stained section slides alone, poorly differentiated tumors are difficult to separate morphologically specially in truecut biopsies specimens. ${ }^{[4]}$ In these situations ancillary techniques like IHC will help in enhancing the accuracy of arriving at the correct diagnosis.

The purpose of the present study is to examine the accuracy in the diagnosis of NSCLC on true-cut biopsy samples first based on morphology and then with IHC by using different relevant markers such as CK5, CK6, CK7, Napsin-A, TTF-1, P63, Synaptophysin and Chromogranin-A and to recommend an optimal panel of IHC markers for precise diagnosis of NSCLC.

\section{Materials and Methods}

A prospective study on true-cut lung biopsies was done in the Department of Pathology, SVIMS, Tirupati over a period of two years and six months from March 2014 to August 2016. The research was approved by the institutional ethics committee. The computed tomography(CT) guided true cut lung biopsies specimens received in $10 \%$ buffered formalin 
were processed, embedded, cut into $4 \mu$ thick sections, stained with H\&E, mounted and histopathologically reported.

A total of 162 biopsies were received out of which 51 cases were excluded from the study (11 non-neoplastic cases, 8 metastatic, 5 sarcomas, 1 lymphoma, 16 small cell Carcinomas and 10 inadequate biopsies) (Table-1). The remaining 111 samples were subjected to immunohistochemical staining with CK5, CK6, CK7, P63, TTF-1, Napsin-A, Synaptophysin, Chromogranin-A. The sections were deparaffinized in xylene and rehydrated through absolute alcohol.

Antigen retrieval in citrate buffer ( $\mathrm{pH} 9$ Lab vision cat\#AP9003) was used after the sections were treated in a microwave at $8 \mathrm{~W}$ for $5-6 \mathrm{~min}$, then at $3 \mathrm{~W}$ for $10 \mathrm{~min}$, and the sections were then left to cool for 20 minutes. Peroxidase and protein blockades were done and the sections were later incubated overnight with the primary antibodies at room temperature using Cytokeratin5(CK5) pre-diluted rabbit monoclonal antibody (EPR24/Rabbit), Cytokeratin 6(CK6) pre-diluted rabbit monoantibody (EP67/Rabbit), Cytokeratin7(CK7) pre-diluted rabbit monoclonal antibody(EPR16/Rabbit), P63 pre-diluted mouse monoclonal antibody (4A4/Mouse), TTF-1 prediluted mouse monoclonal antibody(8G7G3/1/Mouse), Napsin- A pre-diluted mouse monoclonal antibody (IP64/Mouse), Synaptophysin pre-diluted polyclonal antibody(rabbit), Chromogranin-A pre-diluted mouse monoclonal antibody (LK2H10/Mouse) from Biogenix company followed by rinsing in PBS (phosphate buffered saline, $\mathrm{pH}$ 7.6). This was followed by incubation with the secondary biotin conjugated antibody for one hour and finally incubation with peroxidase conjugated streptavidin for another hour. Diaminobenzidine tetrachloride (DAB) was added and kept for 25 minutes, and then counterstained in Haematoxylin, followed by dehydration, clearing and mounting. Positive control sections were prepared from squamous cell carcinoma of nasopharynx for CK5, CK6 and P63, papillary carcinoma of thyroid for TTF1, lung adenocarcinoma for Napasin-A, paraganglioma for synaptophysin and chromogranin-A. Negative controls were done by excluding primary antibody and its replacement with PBS. Presence $(\geq 1 \%$ positivity in lesional cells) and absence of immunoreactivity in tumor cells were given $1 \& 0$ points respectively. ${ }^{[5]}$

Statistical Analysis: Each case data was entered in a Microsoft Excel 2007, spread sheet (Microsoft Corp, Redmond, WA). Fisher's exact test (SPSS20 VERSION) was used for analysis of contingency tables with significance level at $\mathrm{p}<0.05$.

\section{Results}

The subjects were aged between 24 years to 85 years. The youngest was a 24 -year-old male and oldest was an 85 -yearold female. Majority of the patients were in $6^{\text {th }}$ decade (41 cases, $37 \%)$ followed by $7^{\text {th }}$ (36 cases, $\left.32.5 \%\right)$ ), $8^{\text {th }}(19$ cases, $17 \%), 5^{\text {th }}\left(10\right.$ cases, $9 \%$ ) and $9^{\text {th }}$ decade (3cases, $2.7 \%$ ) and one each $(0.9 \%)$ in the $3^{\text {rd }}$ and $4^{\text {th }}$ decades. The male: female ratio is $3.5: 1$. The mean age group is $61.66 \pm 10.03$ years.

Out of 111 cases, adenocarcinomas were $80(72 \%)$ with maximum cases being in the 6th decade and squamous cell carcinomas were $31(28 \%)$ with maximum cases being in the $7^{\text {th }}$ decade (12), with male preponderance seen in both adenocarcinoma (M: F-3:1) and squamous cell carcinoma (M: F-7:1). Initial diagnosis on H\&E of adenocarcinoma, 62 cases, and squamous cell carcinoma, 14 cases, were confirmed after IHC. Out of 21 cases initially diagnosed as NSCLC (unclassifiable) on H\&E, 12 cases were diagnosed as adenocarcinoma and 9 cases were diagnosed as squamous cell carcinoma after IHC. Out of 14 cases which were initially diagnosed as poorly differentiated carcinoma on H\&E, 6 cases were diagnosed as adenocarcinoma and 8 cases were diagnosed as squamous cell carcinoma after IHC (Table 2).

The individual IHC marker positivity among the adenocarcinoma cases $(\mathrm{n}=80)$ is given in Table 3. Among the 80 cases, 60 cases were positive for CK7, Napsin-A, and TTF-1, 11 cases were positive for CK 7 and Napsin-A in, 5 cases were positive for CK 7 and TTF- 1 and 2 cases were positive for TTF-1 and Napsin-A.

The individual IHC marker positivity among the squamous cell carcinoma cases $(n=31)$ is given in Table 4. Among the 31 cases, 22 cases were positive for CK5, CK6, P63, 2 cases were positive for CK6 and P63, 1 case each for CK5, P63 and CK5, CK6. P63 alone was positive in 3 cases and 2 cases were positive only for CK5.

\section{Discussion}

In India, lung cancer constitutes 6.9 per cent of all new cancer cases and 9.3 per cent of all cancer related deaths in both sexes. It is the most common cause of cancer related mortality in men. ${ }^{[6]}$ Surgery is the treatment of choice for early stage of lung cancer and radiotherapy with or without chemotherapy is the treatment for inoperable stage I, stage II and stage III cases ${ }^{[7]}$ The overall 5 -year survival rate of lung cancer is dismal with approximately 15 per cent in developed countries and 5 per cent in developing countries. ${ }^{[7]}$ In the Western countries and most of the Asian countries adenocarcinoma presentation has surpassed the previous common observations of squamous cell carcinoma. 
Table 1: Total lung biopsy specimens received.

\begin{tabular}{|c|c|c|}
\hline \multirow{2}{*}{ INCLUDED CASES } & LESIONS & NUMBER OF CASES (n) \\
\hline \multirow{2}{*}{ EXCLUDED CASES } & Non-small cell lung carcinomas & 111 \\
\cline { 2 - 3 } & Non-neoplastic lesions & 5 \\
\cline { 2 - 3 } & Metastatic lesions & 11 \\
\cline { 2 - 3 } & Sarcomas & 16 \\
\cline { 2 - 3 } & Lymphomas & 10 \\
\cline { 2 - 3 } & Small cell carcinomas & 162 \\
\cline { 2 - 3 } & Inadequate & 16 \\
\hline
\end{tabular}

Table 2: The initial diagnosis made on H\&E stained slides and final diagnosis made after IHC.

\begin{tabular}{|c|c|c|c|}
\hline \multicolumn{2}{|c|}{ INITIAL DIAGNOSIS [H\&E] } & \multicolumn{2}{c|}{ FINAL DIAGNOSIS AFTER IHC } \\
\hline DIAGNOSIS & NUMBER OF CASES (n) & ADENOCARCINOMA & $\begin{array}{c}\text { SQUAMOUS CELL } \\
\text { CARCINOMA }\end{array}$ \\
\hline Adenocarcinoma & 62 & 62 & 0 \\
\hline $\begin{array}{c}\text { Squamous cell carcinoma } \\
\text { Non-small cell lung } \\
\text { carcinoma unclassifiable }\end{array}$ & 14 & 0 & 9 \\
\hline $\begin{array}{c}\text { Poorly differentiated } \\
\text { carcinoma }\end{array}$ & 21 & 12 & 8 \\
\hline Total & 14 & 6 & 31 \\
\hline
\end{tabular}

Table 3: Positivity and negativity including percentage of IHC markers in adenocarcinoma.

\begin{tabular}{|c|c|c|c|c|c|c|c|c|}
\hline & CK5 & CK6 & P63 & CK7 & TTF1 & NAPSIN-A & SYN & CHROM A \\
\hline POSITIVE n(\%) & $12(15 \%)$ & $15(18.75 \%)$ & $7(8.75 \%)$ & $76(95 \%)$ & $60(75 \%)$ & $63(78.75 \%)$ & $5(6.25) \%$ & $0(0 \%)$ \\
\hline NEGATIVE n(\%) & $68(85 \%)$ & $65(81.25 \%)$ & $73(91.25 \%)$ & $4(5 \%)$ & $20(25 \%)$ & $17(21.25 \%)$ & $75(93.75 \%)$ & $0(100 \%)$ \\
\hline
\end{tabular}

Table 4: Positivity and negativity including percentage of IHC markers in squamous cell carcinoma.

\begin{tabular}{|c|c|c|c|c|c|c|c|c|}
\hline & CK5 & CK6 & P63 & CK7 & TTF1 & NAPSIN-A & SYN & CHROM-A \\
\hline POSITIVE n(\%) & $26(84 \%)$ & $25(81 \%)$ & $28(90 \%)$ & $9(35.5 \%)$ & $2(6.5 \%)$ & $0(0 \%)$ & $2(6.5 \%)$ & $0(0 \%)$ \\
\hline NEGATIVE n(\%) & $5(16 \%)$ & $6(19 \%)$ & $3(10 \%)$ & $20(65 \%)$ & $29(93.5 \%)$ & $31(100 \%)$ & $29(93.5 \%)$ & $31(100 \%)$ \\
\hline
\end{tabular}




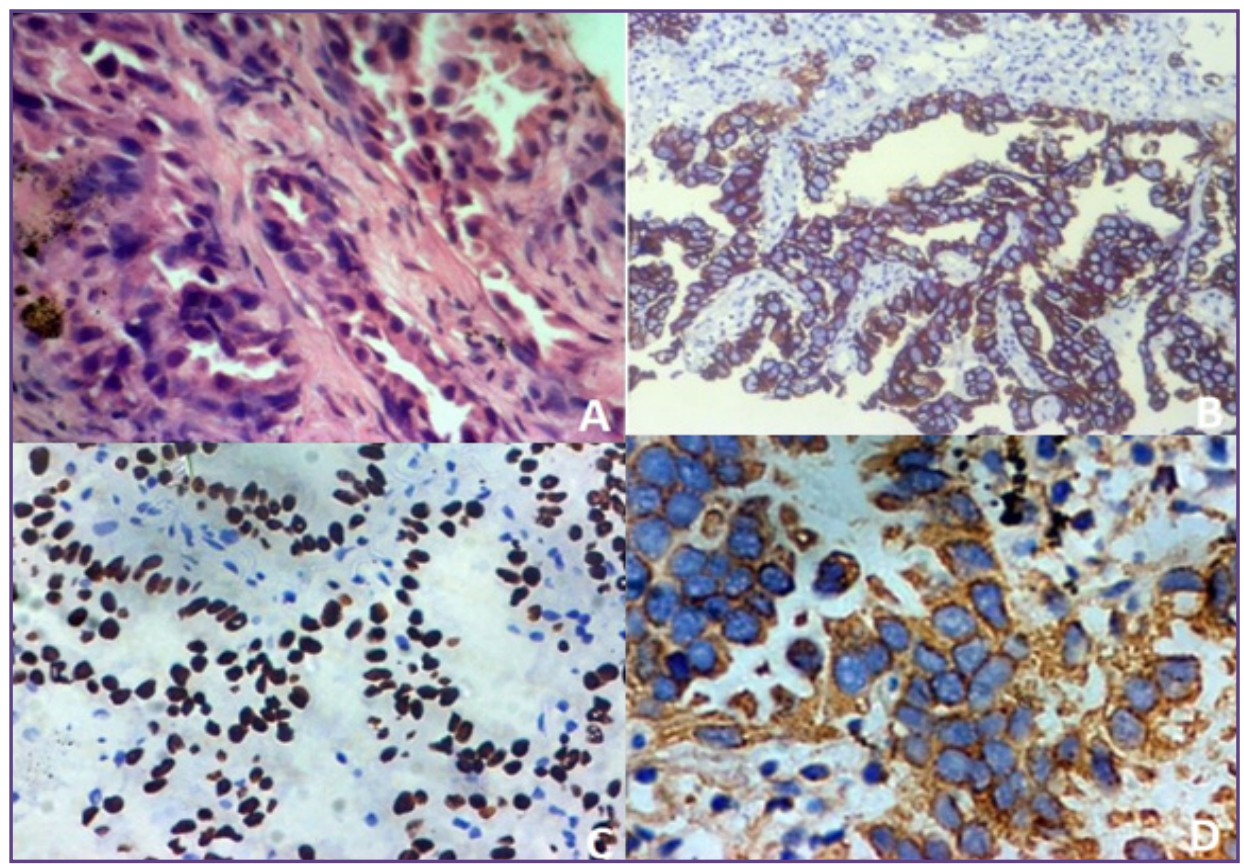

Fig. 1A: Photomicrograph showing adenocarcinoma with cuboidal cells with mild nuclear pleomorphism arranged in the form of glandular exhibitions-true-cut lung biopsy(H\&E20X) Fig1B: Photomicrograph showing diffuse cytoplasmic positivity of cytokeratin7 in the lesional cells of true-cut lung biopsy diagnosed as adenocarcinoma after ihc.(IHC- 20X).Fig 1C: Photomicrograph showing diffuse nuclear positivity of TTF-1 in the lesional cells of true-cut lung biopsy diagnosed as adenocarcinoma after ihc.(IHC- 20X).Fig1D: Photomicrograph showing diffuse granular cytoplasmic positivity of Napsin-A in the lesional cells of true-cut lung biopsy diagnosed as adenocarcinoma after ihc.(IHC- 20X).

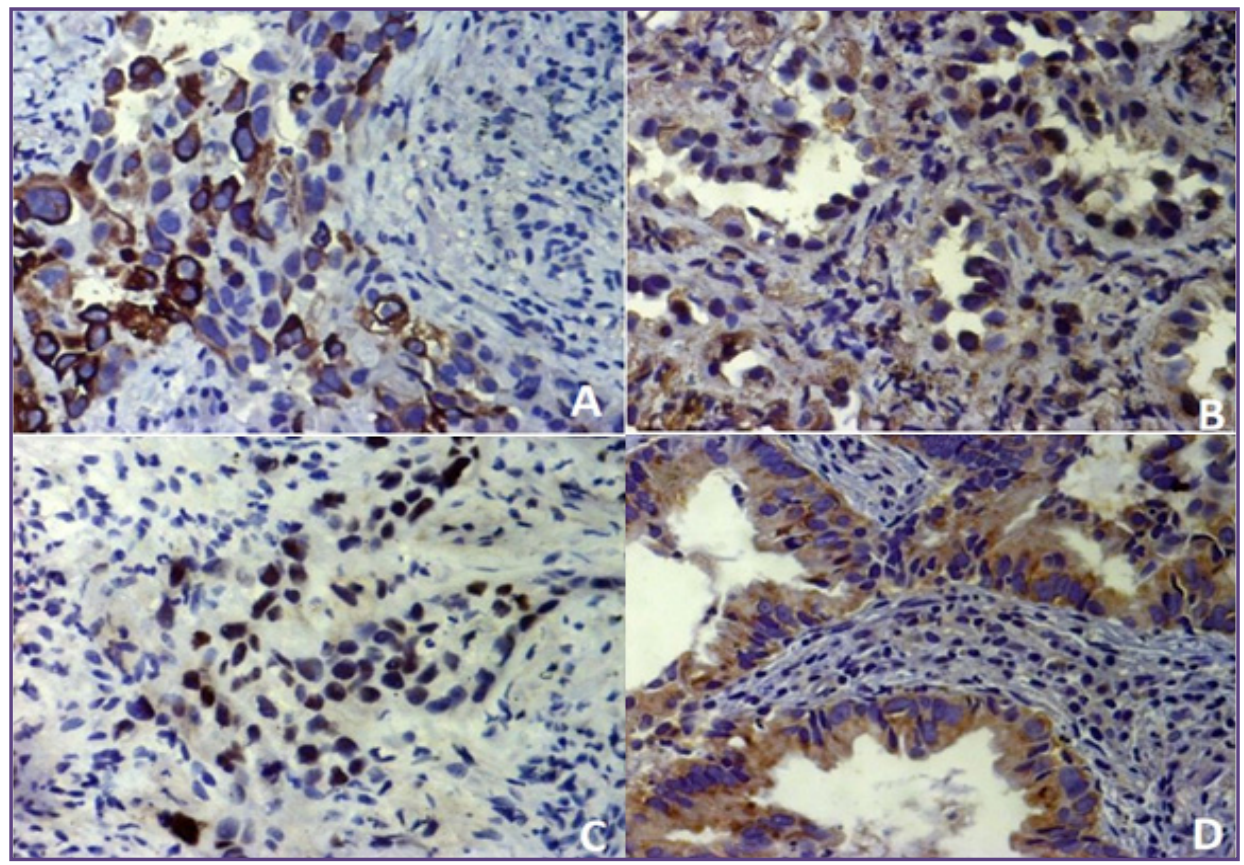

Fig. 2A: Photomicrograph showing focal cytoplasmic CK5 positivity in adenocarcinoma-true-cut lung biopsy. (IHC-20X) Fig2B : Photomicrograph showing focal cytoplasmic CK6 positivity in adenocarcinoma-true-cut lung biopsy. (IHC-20X) Fig2C: Photomicrograph showing focal nuclear P63 positivity in adenocarcinoma-true-cut lung biopsy. (IHC-20X)Fig 2D: Photomicrograph showing focal cytoplasmic Synaptophysin positivity in adenocarcinoma-true-cut lung biopsy. (IHC-20X). 


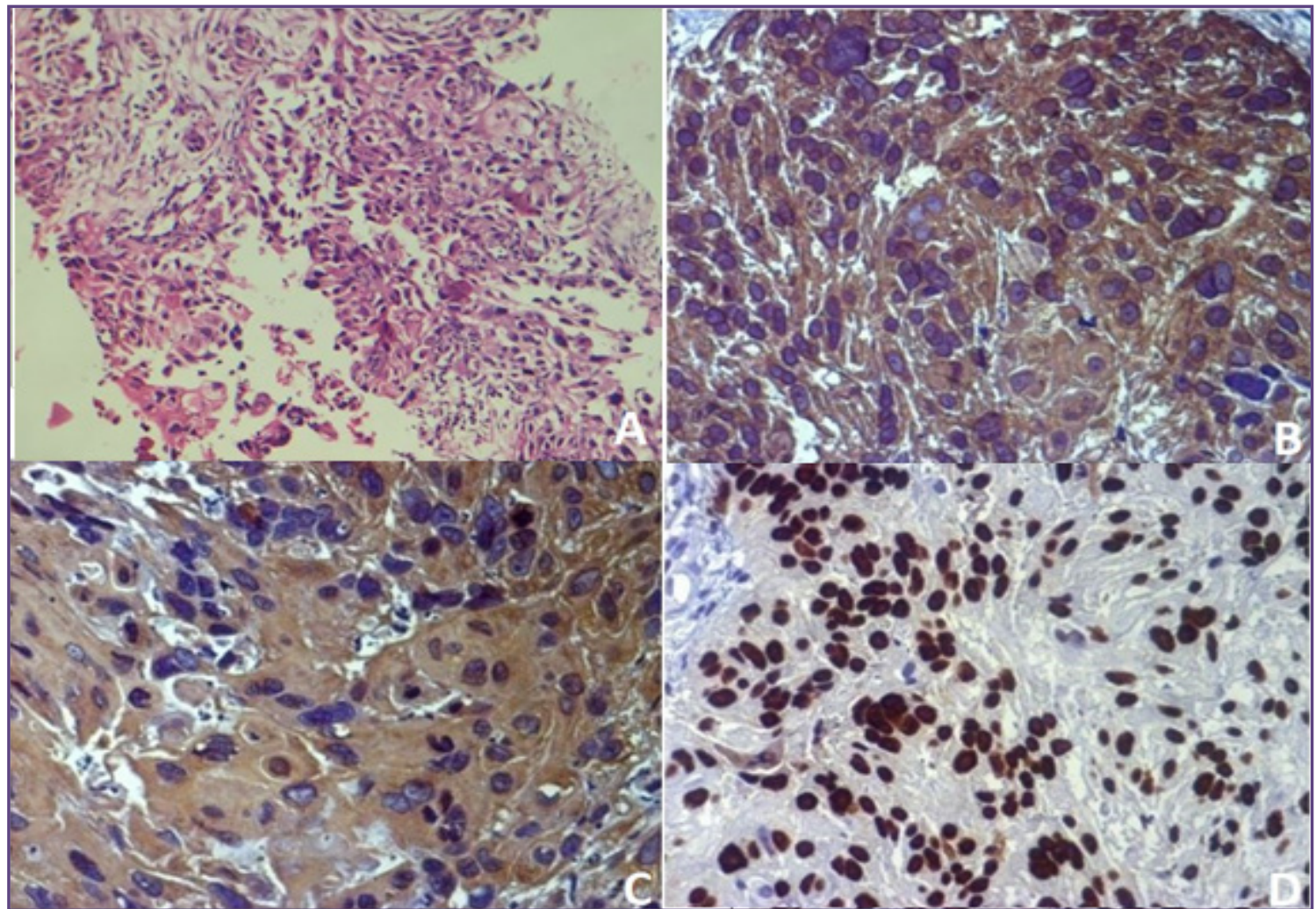

Fig. 3A: Photomicrograph showing squamous cell carcinoma with islands of tumor cells with pleomorphic hyperchromatic nuclei and abundant eosinophilic cytoplasm-true-cut lung biopsy(H\&E 20X)Fig3B: Photomicrograph showing diffuse cytoplasmic positivity of CK5 in the lesional cells of true-cut lung biopsy diagnosed as squamous cell carcinoma after ihc. (IHC- 20X).Fig3C: Photomicrograph showing diffuse cytoplasmic positivity CK6 in the lesional cells of true-cut lung biopsy diagnosed as squamous cell carcinoma after ihc.(IHC- 20X).Fig3D: Photomicrograph showing diffuse nuclear positivity of P63 in the lesional cells of true-cut lung biopsy diagnosed as squamous cell carcinoma after ihc.(IHC- 20X).

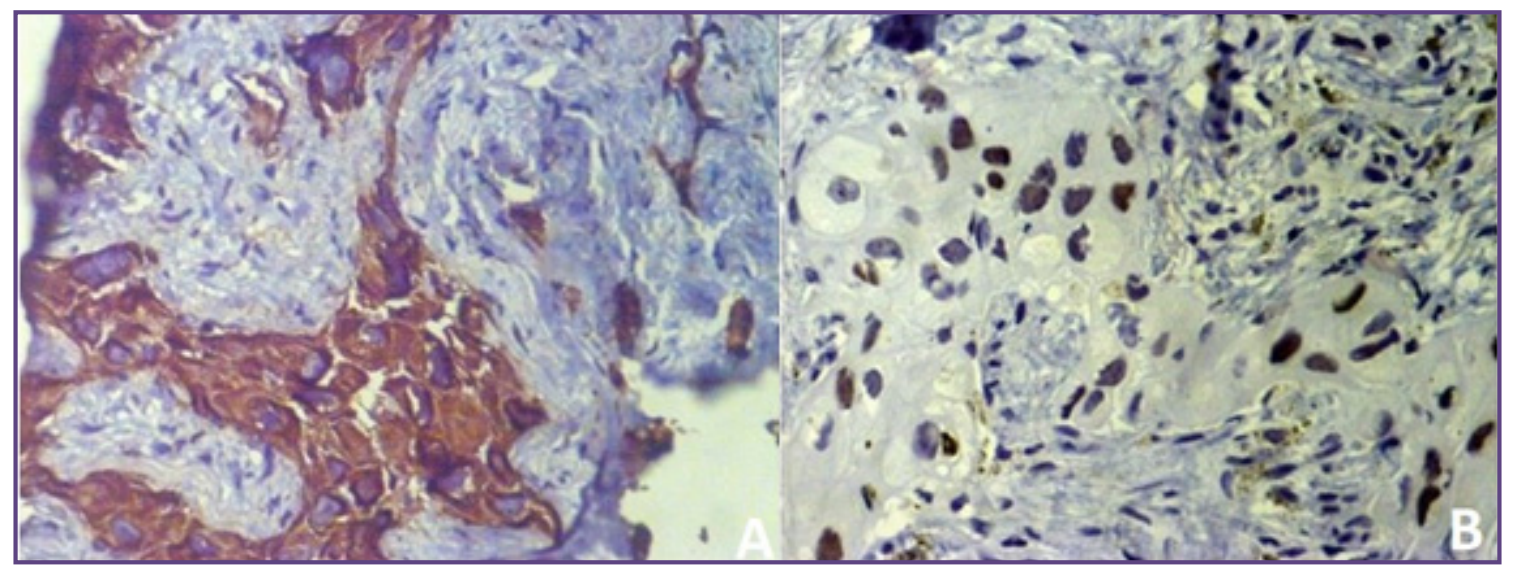

Fig. 2A: Photomicrograph showing focal cytoplasmic CK5 positivity in adenocarcinoma-true-cut lung biopsy. (IHC-20X) Fig2B : Photomicrograph showing focal cytoplasmic CK6 positivity in adenocarcinoma-true-cut lung biopsy. (IHC-20X) Fig2C: Photomicrograph showing focal nuclear P63 positivity in adenocarcinoma-true-cut lung biopsy. (IHC-20X)Fig 2D: Photomicrograph showing focal cytoplasmic Synaptophysin positivity in adenocarcinoma-true-cut lung biopsy. (IHC-20X). 
${ }^{[8,9,10]}$ Jindal \& Behera have reported that Indian patients present almost 15-20 years earlier, compared to Western population, that is in the $5^{\text {th }}$ or $6^{\text {th }}$ decades of life. ${ }^{[11]}$

In the present study among the 111 primary lung carcinomas studied, the male: female ratio is $3.5: 1$, with majority of the patients in $6^{\text {th }}$ decade similar to observations of Koul et al, ${ }^{[12]}$ Sheikh et al ${ }^{[13]}$ Jindal and Behera. ${ }^{[11]}$ There are studies reporting adenocarcinoma in younger age both in men and women. ${ }^{[14]}$ Histological subtypes in the final diagnosis of present study include adenocarcinoma and squamous cell carcinoma, with adenocarcinomas being the most common, which is in agreement with recent Indian studies. ${ }^{[9,16]}$ and also in contrast to some Indian studies. ${ }^{[10,17]}$

IHC IN Adenocarcinoma: CK7, an intermediate filament protein, weighs about $54 \mathrm{KD}$, is a type 2 keratin of simple non-keratinizing epithelium, found in breast, lung, ovarian, pancreatico-biliary malignancies and transitional cell carcinomas. In the present study, out of 80 cases of adenocarcinoma (Fig-1A), CK7 is positive in 76 cases (95\%) (Fig-1B) similar to the findings of Gurda et al $(93.8 \%)^{[18]}$ and Sterlacci et al ${ }^{[19]}(97.2 \%)$. Studies have also reported $100 \%$ positivity ${ }^{[20,21]}, 80.18 \%{ }^{[22]}$ and $84 \%$ ${ }^{[14]}$ positivity. In present study, CK 7 is negative in 4 cases, among them 2 cases are negative for all the 8 markers, but morphology of the tumour revealed glandular exhibitions. Hence the diagnosis of adenocarcinoma which was made on morphology could not be excluded in these two cases.

TTF-1 is a nuclear transcription factor that is expressed in epithelial cells of lung and thyroid. In the lung, it regulates the expression of genes involved in the production of surfactant. TTF-1 is also immunoreactive in other tumors like thyroid neoplasms, breast adenocarcinoma, gastro intestinal carcinoma, small cell carcinoma, carcinoid and possibly controversially in squamous cell carcinoma of lung. Kargi et al reported that in small cell carcinoma sometimes it will be positive for TTF-1 and negative for P63. ${ }^{[23]} \mathrm{In}$ present study, TTF-1 is positive in 60 adenocarcinoma cases $(75 \%)$ (Fig-1C) which is higher when compared to the studies done by Kargi et al (40\%) ${ }^{[23]}$, Sainz et al (63\%) ${ }^{[24]}$ and in agreement with Modi et al ${ }^{[14]}$ (74\%), Bishop JA et al $(73 \%) \cdot{ }^{[25]}$ Higher percentage of positivity has been reported by Mukopadhyay $\mathrm{S}$ et al $(80 \%)^{[26]}$, Zhao et al $(80 \%)^{[21]}$, Gurda et al $(84.5 \%)^{[18]}$, Sterlacci et al $(83.5 \%)$ ${ }^{[19]}$ Yaman et al ${ }^{[27]}(85 \%)$, Burnnstrom et al ${ }^{[20]}(92 \%)$ and Xuxy et al (94.59\%). ${ }^{[2]}$ The reason for above variability in positivity for TTF-1 could be because TTF-1 will be more positive in better differentiated adenocarcinoma. ${ }^{[28]}$ Of 20 cases of adenocarcinomas which were TTF-1 negative, 17 cases were CK7 positive, 7 cases were positive for Napsin-A, 3 cases were CK 7 negative and 11 cases were negative for Napsin-A.
Napsin -A (35 KD) protein expressed in type II pneumocytes, alveolar macrophages and renal tubular cells. It is an aspartic protease involved in the posttranslational modification of surfactant protein $B$ in type II pneumocytes. The expression of Napsin-A has been shown to be transcriptionally regulated by TTF1. Napsin-A is particularly useful in identifying poorly differentiated adenocarcinoma of lung which is negative for TTF-1and it will be consistently negative for squamous cell carcinoma. ${ }^{[29]}$ Thus TTF-1 negative and $\mathrm{P} 63$ negative NSCLC can be diagnosed as adenocarcinoma. We found Napsin-A is positive in 63(78.75\%) cases(Fig-1D) which is nearly similar to studies done by Bishop JA et al ${ }^{[25]}(83 \%)$ and Sainz et al ${ }^{[24]}$ (74\%). These findings were different from the positivity found by Zhao et al ${ }^{[21]}$ (64\%), Gurda et al ${ }^{[18]}(92 \%)$ and Burnnstrom et al ${ }^{[20]}(92 \%)$. In our study Napsin-A positivity (78.75\%) is higher when compared to TTF-1 positivity (75\%). Study by Gurda et al ${ }^{[18]}$ reported addition of Napsin-A to routine practice coincided with an increase in diagnosis of adenocarcinoma from $14 \%$ to $36 \%$ with a concurrent decrease in NSCLC-unclassifiable. Limitations of Napsin-A is that it will be positive in some cases of small cell carcinoma and carcinoid, renal cell carcinoma and it also stains pulmonary macrophages. In 17 cases which are Napsin-A negative, 5 cases showed positivity for CK7 and TTF-1, 15 cases showed positivity for only CK7.

CK5 a basic high molecular weight polypeptide (58KD), is expressed in neoplasms of epithelial origin, including squamous cell carcinoma, mesothelial carcinoma and urothelial carcinoma. CK5 negative staining together with TTF-1 negativity and P63 diffuse positivity will be enough to support diagnosis of squamous cell carcinoma. We found CK5 is positive in $12(15 \%)$ cases(Fig-2A) unlike the studies done by Burnnstrom et al ${ }^{[20]}(1 \%)$ and Kargi et al ${ }^{[23]}(20 \%)$ whereas Zhao et al ${ }^{[21]}$ and Mukopadhyay S et al ${ }^{[26]}$ did not show CK5 positivity in adenocarcinoma. The reason for this could be that $30 \%$ of pulmonary adenocarcinoma can show focal variable immunostaining for CK5. ${ }^{[30]}$ Out of 12 cases of adenocarcinomas showing positivity for CK5, 7 cases showed positivity for CK7, TTF-1 \& Napsin-A, 2 cases showed negativity for only TTF-1, 1 case showing negativity for both TTF-1 and Napsin- A and 1 case was negative for CK7.

CK6 a basic high molecular weight polypeptide (56KD) is expressed in neoplasms of epithelial origin, including squamous cell carcinoma, mesothelial carcinoma and urothelial carcinoma. We found CK-6 is positive in $15(18.75 \%)$ cases(Fig-2B). Zhao et al ${ }^{[21]}$ did not reveal any positivity for CK6 in adenocarcinomas, reason could be that $30 \%$ of pulmonary adenocarcinoma can show focal 
variable immunostaining for CK6. ${ }^{[30]}$ Out of 15 cases showing positivity for CK6, 9 cases showed positivity for CK7, TTF-1 and Napsin-A. Positivity for CK7 alone was seen in 2 cases. 3 cases were positive for CK $7 \&$ Napsin-A. 3 cases were negative for TTF- 1 alone and 2 cases were negative for both TTF-1 and Napsin-A.

Human TP63 gene is located on the chromosome 3q2729 and the expression of the gene produces the full-length protein P63 and the truncated P40. P63 can be detected in benign bronchial stem cells and in neoplastic cells with evidence of squamous differentiation. ${ }^{[18]}$ P63 will be positive in metastatic urothelial carcinoma, myoepithelial neoplasms, lymphoma and sarcoma. P63 can be expressed in $21.7 \%$ of adenocarcinoma. ${ }^{[18]}$ A $10-65 \%$ positivity of P63 in adenocarcinoma was reported ${ }^{[23]}$ where it shows weak and focal expression in contrast to strong and diffuse expression in squamous cell carcinoma. It has been shown that P63 expression will be seen in poorly differentiated adenocarcinoma which are negative for TTF-1 and Napsin-A. ${ }^{[18]}$ Several studies showed that P63 is consistently positive in lung squamous cell carcinoma irrespective of the degree of differentiation. ${ }^{[26]}$ In present study, P63 is positive in $7(8.75 \%)$ cases(Fig-2C), similar to Sterlacci et al (7\%) ${ }^{[19]}$ and Mukopadhyay S et al (10\%). ${ }^{[26]}$ Studies done by Burnnstrom et al ${ }^{[20]}(26 \%)$ and Zhao et al $^{[21]}(16 \%)$ revealed high percentage of positivity. Out of 7 cases of adenocarcinomas showing P63 positivity, 6 cases showed positivity for CK7, TTF-1 and Napsin-A and in one case patchy positivity for $\mathrm{P} 63$ was seen in association with diffuse TTF-1 positivity. Yaman et al [27], Modi et al [14] and Kargi et al ${ }^{[23]}$ did not find $\mathrm{P} 63$ positivity.

Synaptophysin in the present study is positive in $5(6.25 \%)$ cases(Fig-2D) which is nearly equal to the study done by Burnnstrom et al ${ }^{[20]}(7 \%)$. Out of 5 cases of adenocarcinoma showing positivity for synaptophysin, 4 cases were positive for CK7, TTF-1, Napsin-A except for 1 case which is negative for TTF-1 and positive for CK7 and Napsin-A.

Chromogranin-A was negative in all of the cases $(0 \%)$ in the present study.

Squamous Cell Carcinoma (Fig-3A) CK5 was found to be positive in $26(84 \%)$ cases(Fig-3B) which is slightly higher than Zhao et al ${ }^{[21]}(81.25 \%)$. Mukopadhyay S et al ${ }^{[26]}$ reported positivity of $73 \%$. Xuxy et al ${ }^{[22]}$, Burnnstrom et al ${ }^{[20]}$ and Sterlacci et al ${ }^{[19]}$, revealed higher percentage of positivity $91.92 \%, 98 \% \& 99.2 \%$ respectively. Gurda et al ${ }^{[18]}$ reported $100 \%$ positivity. Out of 31 cases of squamous cell carcinomas, 26 cases showed positivity for CK5 and 5 cases were negative. All the CK5 negative cases are positive for P63 and only 2 CK5 negative cases were positive for CK6.
CK6 is positive in $25(81 \%)$ cases(Fig-3C), similar to Zhao et al ${ }^{[21]}(81.25 \%)$. In the 6 cases negative for CK6, 2 cases were positive for CK5 alone, 2 cases for P63 alone, 1 case for both CK5 and P63 and 1 case for P63 and CK7. Studies by Sterlacci et al ${ }^{[19]}(99.2 \%)$, Xuxy et al ${ }^{[22]}(91.92 \%)$ and Mukopadhyay S et al ${ }^{[26]}$ (73\%) reported varying percentages of positivity. Gurda et al ${ }^{[18]}$ reported $100 \%$ positivity.

P63 is positive in 28(90\%) cases(Fig-3D) in our study similar to Yaman et al ${ }^{[27]}(87.5 \%)$, Gurda et al ${ }^{[18]}(91.75 \%)$, Xuxy et al ${ }^{[22]}(96.95 \%)$, Burnnstrom et al ${ }^{[20]}$ (97\%) and Sterlacci et al $(99.2 \%) .{ }^{[19]}$ Mukopadhyay $\mathrm{S}$ et al ${ }^{[26]}$ and Zhao et al ${ }^{[21]}$ reported $100 \%$ positivity. Kargi A et al ${ }^{[23]}$ and Modi et al ${ }^{[14]}$ revealed $61 \%$ and $82.05 \%$ positivity. Among the 3 P63 negative cases one was positive for CK5 and CK6 and 2 were positive for only CK5.

CK-7 is positive in $11(35.5 \%)$ cases in the present study (Fig-4A). Out of 11 cases showing positivity for CK7, 8 showed positivity for CK5, CK6 \& P63,1 case showed positivity for CK5 and CK6 and 2 cases showed positivity for only P63.Varying percentages are reported by Sterlacci et al ${ }^{[19]}(23.5 \%)$, Burnnstrom et al ${ }^{[20]}(46 \%)$, Gurda et al ${ }^{[18]}(50 \%)$, Modi et al ${ }^{[14]}(57 \%)$ and Zhao et al ${ }^{[21]}(62.5 \%)$.

TTF-1 is positive in $2(6.5 \%)$ cases(Fig-4B) which is higher than Sterlacci et al ${ }^{[19]}(3.4 \%)$, In these 2 cases of squamous cell carcinoma, CK5, CK6 and P63 showed positivity. Modi et al [14], Kargi et al [23], Mukopadhyay S et al [26], Burnnstrom et al ${ }^{[20]}$ and Zhao et al ${ }^{[21]}$ did not find positivity for TTF-1.

Napsin-A is negative in all the cases $(0 \%)$ similar to Zhao et al ${ }^{[21]}(0 \%)$. Burnnstrom et al ${ }^{[20]}$ found $5 \%$ positivity.

Synaptophysin is positive in $2(6.5 \%)$ cases, higher than Burnnstrom et al ${ }^{[20]}(2 \%)$.In these 2 cases CK5, CK6 and P63 were positive. In 1 case in addition to these 3 markers, CK7 positivity is also seen.

Chromogranin-A is negative in all the cases $(0 \%)$ in the present study. Although not common, positive neuroendocrine markers do not rule out the diagnosis because few cases of adenocarcinomas, squamous cell carcinomas and non-neuroendocrine large cell carcinomas showed positivity for neuroendocrine markers in other studies.

In 12 cases of NSCLC- unclassifiable which turned out to be adenocarcinomas after IHC,5 cases showed positivity for CK7, TTF-1 and Napsin-A, 2 cases positive for CK7, TTF-1, 2 cases positive for CK7 and Napsin-A and 3 cases are positive for TTF-1 and Napsin-A. In 6 cases of poorly differentiated carcinomas which turned out to be 
adenocarcinomas after IHC, 5 cases showed positivity for CK7, TTF1 and Napsin-A and 1 case showed positivity for CK7 and TTF1. In 9 cases of NSCLC- unclassifiable which turned out to be squamous cell carcinomas after IHC, 5 cases showed positivity for CK5, CK6 and P63, 1 case positive for CK5 and P63, 1 case positive only for P63 and 2 cases positive for CK5 and CK6. In 8 cases of poorly differentiated carcinomas which turned out to be squamous cell carcinomas after IHC, 6 cases showed CK5and P63 positivity, 2 cases showed only P63 positivity.

CK5, CK6 and P63 $(\mathrm{p}<0.001)$ may help in detecting squamous cell carcinoma and CK7, TTF-1, Napsin$\mathrm{A}(\mathrm{p}<0.001)$ may help in detecting adenocarcinoma. Synaptophysin $(\mathrm{p}=1.00)$ is not helpful in detecting either adeno and squamous cell carcinoma.

\section{Conclusion}

A panel of IHC markers are helpful to differentiate adenocarcinoma and squamous cell carcinoma on small true-cut lung biopsies which can influence the personalized treatment of the patient. CK5, CK6 and P63 help in detecting squamous cell carcinoma, CK7, TTF-1 and Napsin-A for detecting adenocarcinoma. In NSCLC-unclassifiable and poorly differentiated carcinoma as diagnosis on $\mathrm{H} \& \mathrm{E}$, we recommend a IHC panel consisting of P63, TTF-1 and Napsin-A for a definitive diagnosis. From our study, we infer that Napsin-A will be negative in squamous cell carcinoma.

\section{Limitations}

Our study had few limitations like small sample size and most of the patients were in advanced stage at the time of presentation. Neither follow up of every patient for clinical improvement, resected lung specimens nor autopsy findings for final histopathology reports were available. Hence sensitivity, specificity, positive predictive value and negative predictive value could not be calculated in the present study.

\section{Acknowledgements}

I acknowledge the technical assistance rendered by senior technologists Mrs Usha Nandini and Mr. Venkata Ramaiah, and assistance in the critical review and editing of the manuscript provided by Dr.M.M.Suchitra, Associate Professor, Department of Biochemistry, SVIMS.

\section{References}

1. Ferlay J, Soerjomataram I, Ervik M, Dikshit R, Eser S, Mahters C, et al. GLOBOCAN 2012 v1.0,Cancr Incidence and Mortality Worldwide: IARC Cancer Base No. 11.Lyon, France; International Agency for Reasearch on cancer; 2013.

2. Ferlay J, Shin HR, Bray F, Forman D, Mathers C, Parkin DM. Estimates of world burden of cancer in 2008. Int. J. Cancer 2010; 127: 2893-2917.
3 Husian Aliya N. The Lung: Tumours. In: Kumar, Abbas,Aster (Eds). Robbins and Cotran Pathologic basis of disease: 9thed.Elsevier ;2015.

4. Grilley-Olson JE, Hayes DN, Moore DT, Leslie KO, Wilkerson MD, Qaqish BF, et al. Validation of interobserver agreement in lung cancer assessment: haematoxylin-eosin diagnostic reproducibility of non-small-cell lung cancer: the 2004 World Health Organisation classification and therapeutically relevant subsets. Arch Pathol Lab Med 2013; 37:32-40.

5. Whithaus K, Fukuoka J, Prihoda TJ et al. Evaluation of napsin-A, cytokeratin 5/6, p63 and thyroid transcription factor 1 in adenocarcinoma versus squamous cell carcinoma of the lung. Arch Pathol Lab Med.2012; 136:155-162.

6. National Cancer Registry Programme. Three Year Report of Population Based Cancer Registries: 2009-2011. Indian Council of Medical Research; 2013. Available from: http:// www.ncrpindia.org, accessed on January 21, 2014.

7. Parkin DM, Bray F, Ferlay J, Pisani P. Global cancer statistics, 2002. CA Cancer J Clin2005; 55: 74-108.

8. Valaitis J, Warren S, Gamble D. Increasing incidence of adenocarcinoma of the lung. Cancer 1981; 47: 1042-6.

9. Malik PS, Sharma MC, Mohanti BK, Shukla NK, Deo S, Mohan A, et al. Clinico-pathological profile of lung cancer at AIIMS: A changing paradigm in India. Asian Pac J Cancer Prev2013; 14: 489-94.

10. Singh N, Aggarwal AN, Gupta D, Behera D, Jindal SK.et al. Unchanging clinico-epidemiological profile of lung cancer in north India over three decades. Cancer Epidemiol2010; 34: 101-4.

11. Jindal SK, Behera D. Clinical spectrum of primary lung cancer: Review of Chandigarh experience of 10 years. Lung India 1990; 8: 94-8.

12. Koul PA et al. "Lung cancer in the Kashmir valley “. Lung India 27.3(2010): 131-137.

13. Sheikh S, et al. "Histopathological pattern of Primary Malignant Lung Tumors Diagnosed in a Tertiary Care Hospital :10 -year study". Asian Pacific Journal of Cancer Prevention 111.5(2010): 1341-1346.

14. Mitesh R.R, Mithul B.M, Ramrao G, Nilkhanthe, Truti S, Dhaval $\mathrm{H}$ et al. A Detailed Study of Seventy Cases of NonSmall Cell Carcinoma of Lung, Immuohistochemical study and its Histo-Cytological Correlation. EC Pulmonology and Respiratory Medicine 2.3 (2016): 113-122.

15. Noronha V, Dikshit R, Raut N, Joshi A, Pramesh CS, George $\mathrm{K}$, et al. Epidemiology of lung cancer in India: focus on the differences between non-smokers and smokers: a singlecentre experience. Indian J Cancer 2012; 49: 74-81.

16. Mondal SK et al. "Computed tomogram guided fineneedle aspiration cytology of lung mass with histological correlation: A study in Eastern India". South Asian Journal of Cancer 2.1(2013): 14-18. 
17. Himanshi D, Ajay Y, Jayanti M. Clinico-morphological and Immunohistochemical profile of Lung Carcinoma Specimens Received at a Tertiary care Centre: Journal Of Medical Science And Clinical Research Vol |[05]| Issue|[06]| Page 23103-23110|| June.

18. Grzegorz TG, Lei Z, Yuting W, Li C, Susan G, William CC et al. Utility of five commonly used immunohistochemical markers TTF1, Napsin-A, CK7, CK5/6 and P63 in primary and metastatic adenocarcinoma and squamous cell carcinoma of the lung: a retrospective study of 246 fine needle aspiration cases. Clinical and Translational Medicine (2015) 4:16.

19. Sterlacci $\mathrm{W}$ et al. Tissue-sparing application of the newly proposed IASLC/ATS/ERS classification of adenocarcinoma of the lung shows practical diagnostic and prognostic impact. Am J ClinPathol 2012 Jun; 137(6): 946-56.

20. Brunnstrom $\mathrm{H}$ et al. Immunohistochemistry in the differential diagnostics of primary lung cancer: an investigation within the Southern Swedish Lung Cancer Study. Am J ClinPathol $2013 \mathrm{Jul} ; 140(1): 37-46$.

21. Zhao $\mathrm{W}$ et al. $\triangle \mathrm{Np} 63, \mathrm{CK} 5 / 6, \mathrm{TTF}-1$ and napsin-A, a reliable panel to subtype non-small cell lung cancer in biopsy specimens. Int J ClinExpPathol2014 Jun 15; 7(7): 4247-53.

22. Xu XY, Yang GY, Yang JH, Li J. Analysis of clinical characteristics and differential diagnosis of the lung biopsy specimens in 99 adenocarcinoma cases and 111 squamous cell carcinoma cases: utility of an immunohistochemical panel containing CK5/6, CK34ßE12, p63, CK7 and TTF-1. Pathol Res Pract 2014 Oct; 210(10): 680-5.
23. Kargi A, Gurel D, Tuna B. The diagnostic value of TTF1, CK5/6 and p63 immunostaining in classification of lung carcinoma. Appl Immunohistochem Mol Morphol. 2007; 15: 415-20.

24. Sainz IM, Fukuoka J, Cagle PT, et al. Napsin-A: a new marker for lung adenocarcinoma is complementary and more sensitive than TTF-1 (thyroid transcription factor-1): evaluation of 967 cases by tissue microarray. Mod Pathol 2008; 21: 349A.

25. Bishop JA, Sharma R, Illei PB. Napsin-A and thyroid transcription factor-1expression in carcinomas of the lung, breast, pancreas, colon, kidney, thyroid, and malignant mesothelioma. Hum Pathol 2010 Jan; 41(1): 20-5.

26. Mukhopadhyay S, Katzenstein AL. Sub-classification of non-small cell lung carcinomas lacking morphologic differentiation on biopsy specimens: Utility of an immunohistochemical panel containing TTF-1, Napsin-A, p63, and CK5/6. Am J SurgPathol 2011 Jan; 35(1): 15-25.

27. Banu Y, Deniz N, Pervin KE, Gursel C, Ali V. Expression of p63, TTF1 and Mapsin in Non-Smallcell Lung Carcinoma and Their Effect on the Prognosis and Differential Diagnosis. Turk Pathol oji Derg 2015,31:163-174.

28. Lau SK, Luthringer DJ, Eisen RN. Thyroid transcription factor-1: a review. Appl Immunohistochem Mol Morphol 2002; 10: 97-102.

29. Hasanovic A, Rekhtman N, Sigel CS, Moreira AL. Advances in the fine needle aspiration cytology for the diagnosis of pulmonary carcinoma. Patholog Res Int. 2011; 8:972-92.37.

30. Clover J, Oates J, Edwards C. Anti-cytokeratin 5/6: a positive marker for epithelioid mesothelioma. Histopathology 1997; 31: $140-43$.

*Corresponding author:

Dr. N. Rukmangadha, Associate Professor, Dept of Pathology, SVIMS, Tirupati 517507, AP, India

Phone: +91 9440674561

Email: nrukmangadha1@rediffmail.com

Financial or other Competing Interests: None. 